Artículo de Opinión

\title{
Tratamientos al final de la vida: cuidados paliativos, sedación terminal, eutanasia y suicidio medicamente asistido (SMA)
}

\author{
Antonio Sacristán Rodea ${ }^{1}$ * y Miguel Ferrari Sanjuan ${ }^{1}$ \\ 1 Profesor asociado de Ciencias de la Salud, Universidad de Alcalá \\ * Autor correspondencia: antonio.sacristan@uah.es \\ DOI: https://doi.org/10.37536/RIECS.2021.6.2.293
}

Recibido: 18/11/2021; Aceptado: 26/11/2021; Publicado: 30/11/2021

\section{Introducción}

Cuando una persona se encuentra al final de su vida, situación no siempre fácil de saber a ciencia cierta, se abre un abanico de posibilidades terapéuticas (a partir de ahora tratamientos al final de la vida) que tienen diferente valoración desde el punto de vista ético, filosófico, religioso, político-social, cultural y, por supuesto, médico. Conviene destacar el papel tan importante que tiene la medicina en el manejo clínico de personas al final de su vida, tratamientos que van desde medidas específicas para enfermedades concretas, aunque no hayan mostrado plenamente su eficacia, hasta otras dirigidas a provocar una muerte rápida, eficaz e indolora.

Los principales tratamientos al final de la vida van a ser: 1) los cuidados paliativos, 2) los tratamientos activos y de soporte como, por ejemplo, la nutrición forzada, los tratamientos antitumorales o la ventilación asistida, 3) la sedación terminal, 4) la eutanasia y, 5) el suicidio medicamente asistido (a partir de ahora SMA), todos ellos incluidos en el concepto global de muerte digna. Algunas de estas alternativas se acompañan, en mayor o menor medida, de aspectos controvertidos porque en su definición y desarrollo entran en juego cuestiones morales y valores esenciales de los individuos, valores como la libertad de elección, la dignidad o la autonomía.

Los cuidados paliativos, ampliamente aceptados a nivel nacional e internacional, ya forman parte de la práctica clínica habitual. Aunque, por el hecho de ser una disciplina relativamente nueva, tiene aspectos pendientes de desarrollo (definición más precisa de los pacientes a incluir en programas de cuidados paliativos, perfil de los profesionales que tienen que hacer esos cuidados, identificación de necesidades a atender, etc.) que pueden suscitar algún tipo de controversia, no es ni con mucho la norma. Hay una cuestión sujeta siempre a discusión y es la relación entre cuidados paliativos y eutanasia pues para algunos son conceptos complementarios mientras que para otros son conceptos antagónicos, donde los cuidados paliativos vendrían a ser una especie de antídoto de la eutanasia.

La sedación terminal o paliativa también ha dejado de ser una cuestión controvertida. Se mantiene una cierta controversia derivada de la consideración de la sedación terminal como una forma de eutanasia lenta [1]. Ha encontrado acomodo legal en las Leyes Autonómicas de Muerte Digna que se han aprobado en la última década en España y actualmente se practica, con mínimas limitaciones, tanto en el medio hospitalario como en la atención domiciliaria.

Se mire por donde se mire, lo que sigue suscitando una enorme controversia es la eutanasia y el suicidio medicamente asistido. El 18 de marzo de 2021, el Congreso de los Diputados aprobó la Ley que regula estas prácticas y con ella se completaba la normativa específica que aborda el conjunto de tratamientos al final de la vida [2]. España es ahora uno de los países con legislación más avanzada en materia de muerte digna, pero la eutanasia y el SMA (asunto en el que la persona decide el cómo y el cuándo de su propia muerte y cuyo centro de discusión versa sobre el significado mismo de la vida) sigue formando parte de intensos debates entre defensores y detractores de la misma. El debate sobre la eutanasia está polarizado en dos extremos: los defensores de la sacralidad de la vida como 
bien supremo y los que consideran que la autonomía y la capacidad de decisión de los individuos en relación a su propia vida, priman frente a cualquier otro valor o principio. Entre estos polos opuestos y difícilmente compatibles, la Ley de eutanasia permite ayudar a morir a las personas que lo soliciten siempre que se den determinadas circunstancias y se cumplan determinados requisitos. Esta situación involucra a un médico, que es quien certifica que se cumplen los requisitos para que una persona pueda acogerse a la Ley y, cuestión clave, es el que proporciona la muerte a los pacientes.

Los cuidados paliativos, la sedación terminal, la eutanasia y el SMA son fenómenos sociosanitarios nuevos, o muy nuevos y, en consecuencia, el planteamiento que se suele hacer de los mismos es muy teórico, con mucha riqueza conceptual y terminológica, no siempre coincidente en su significado y que en muchas ocasiones ensombrece más que ilumina el debate. En consecuencia, gran parte de este trabajo se centra en la definición y clarificación conceptual de los diferentes términos que van a aparecer en el mismo.

\section{El manejo del final de la vida en el mundo actual: dignidad, muerte digna y autonomía}

El interés en el debate de los tratamientos al final de la vida se explica, en gran parte, por los importantes cambios sociales y médicos relacionados con el hecho de enfermar y con el aumento en la duración de la vida que se han producido a lo largo del siglo pasado. Mientras que durante la primera mitad del siglo XX la enfermedad fue un fenómeno de corto alcance ya que la mayoría de las personas morían jóvenes y por procesos agudos, a partir de la segunda mitad mejoró notablemente el control de las enfermedades, incluido el cáncer, y se produjo un aumento notable en la esperanza de vida. Este aumento en la cantidad de la vida está en relación con diferentes factores: disponibilidad de tratamientos más efectivos, avances ligados a la revolución tecnológica y mejora en los determinantes en salud (medioambientales, laborales, hábitos de vida, ...). De hecho, en España, la esperanza de vida al nacer en 1940 era de solo 62,10 años mientras que en 2020 está en 82,34 años, de acuerdo a los datos provisionales facilitados por el Instituto Nacional de Estadística en agosto de 2021 [3]. Pero para algunos autores “... a medida que la duración promedio de la vida aumentaba, también lo hacia la cantidad de enfermedad. Una vida más larga implica una vida marcada por una mayor incidencia de enfermedades, y una mayor duración de las enfermedades una vez que ocurren: el precio de una vida más larga ha sido una vida más enferma. La línea entre extender una vida y extender una muerte se ha hecho casi invisible...porque la medicina encuentra cada vez más difícil localizar la línea entre el vivir y el morir, y así saber cuándo detener el tratamiento por considerarlo inútil" [4].

Los últimos datos de mortalidad por causas específicas proporcionados por el INE [5] en año 2019 indican que la mayoría de las muertes se deben a procesos crónicos, degenerativos y ligados al propio envejecimiento, en los que es posible establecer una historia natural que permite hacer un pronóstico en tiempo y forma, es decir, sabemos lo que va a ocurrir. Este conocimiento va a permitir cambiar los objetivos terapéuticos en la fase final de algunas enfermedades y dirigirlos a un final de vida sin dolor y con el menor sufrimiento posible. Es en este contexto en el que términos como muerte digna o muerte natural, que engloban derechos como la eutanasia, la sedación terminal o los cuidados paliativos, han cobrado una importancia extraordinaria.

Desde un punto de vista médico, en los últimos años se ha pasado, prácticamente sin solución de continuidad, de un modelo paternalista en el que el médico ocupaba el centro del sistema y que no dejaba a sus pacientes ni tan siquiera rechazar o suspender un tratamiento, por ineficaz que resultase, a otro altamente medicalizado en el que, en teoría, es el enfermo el que ocupa el centro y en el que cualquier actuación médica debe regirse por el principio de autonomía, por su derecho a la información, por su participación en el proceso de toma de decisiones y por la posibilidad de otorgar su consentimiento para que se realice cualquier intervención [6]. Aunque en la práctica asistencial coexisten ambos modelos, es difícil aceptar opciones médicas en las que la autonomía del paciente y los otros derechos señalados no ocupen un lugar destacado. Ahora los pacientes pueden elegir y este hecho hace que la relación entre autonomía y muerte digna, incluyendo los cuidados paliativos o la eutanasia, cobre todo su sentido y sirva como garante para que en un momento difícil como es el final de la vida, la dignidad de los pacientes "ni se arrincone ni se menosprecie". 
Antes de entrar a conceptualizar y definir los cuidados paliativos, la sedación terminal o la eutanasia, es necesario hacer referencia a un asunto clave en el abordaje de los tratamientos al final de la vida, el alivio del sufrimiento. Estos tratamientos difieren en fondo y forma en su aplicación y desarrollo, pero todos coinciden en un objetivo que es, precisamente, aliviar el sufrimiento.

\section{Alivio del sufrimiento}

El sufrimiento, concepto difícil de definir, ha generado en la actualidad una enorme demanda social y médica que obliga a considerar la necesidad de su tratamiento. Los pacientes y sus familias insisten de manera recurrente en deseos que ya forman parte de la realidad asistencial del día a día: "yo lo que no quiero es sufrir, no tener dolor" o "yo lo que quiero es que no sufra, que no tenga dolor". No obstante, esta exigencia tan generalizada no ha eliminado la duda acerca de en qué medida la obligación de aliviar el sufrimiento es absoluta.

Sabemos que el sufrimiento es un sentimiento distinto al dolor (más físico y objetivo) y que se vincula con aspectos emocionales, existenciales, vitales $\mathrm{y}$, por tanto, subjetivos. $\mathrm{Y}$ en algunos casos, ajenos a los fines de la medicina. En términos generales, podrían establecerse dos niveles de sufrimiento [4]. Un primer nivel, donde el sufrimiento está ligado al miedo, la incertidumbre o la angustia del paciente para hacer frente a su enfermedad y un segundo nivel, en el que el sufrimiento afecta al significado mismo de la vida. En este último caso, el sufrimiento tiene que ver más con cuestiones filosóficas o religiosas que psicológicas o físicas y, en consecuencia, coloca al médico en un lugar distinto. Mientras que en el primer nivel el médico, a través del tratamiento del dolor, de la provisión de cuidados, de la comunicación y la colaboración con la familia y amigos, debe hacer todo lo posible para disminuir el sufrimiento del paciente, su posición en el segundo supuesto cambia porque es cuestionable que la medicina pueda solucionar esos problemas.

\section{Dignidad, muerte digna y autonomía}

La gestión del final de la vida en la actualidad solo puede entenderse, en términos generales, con el reconocimiento de una nueva concepción del objetivo de la vida en sociedades de consumo, que descansa en dos pilares fundamentales: la reivindicación de la autonomía de las personas y el concepto de dignidad. En un plano más particular, no es posible hablar de tratamientos al final de la vida sin una consideración previa de valores y principios como el de dignidad, muerte digna o autonomía de la persona.

Según el diccionario de la RAE, la dignidad, o «cualidad de digno» (del latín, grandeza), hace referencia "al valor inherente al ser humano por el simple hecho de serlo, en cuanto ser racional, dotado de libertad... no se trata de una cualidad otorgada por nadie, sino consustancial al ser humano... no depende de ningún tipo de condicionamiento ni de diferencias étnicas, de sexo, de condición social o de cualquier otro tipo..."

El reconocimiento jurídico de la dignidad personal aparece en la Declaración Universal de Derechos Humanos aprobada en 1948 que señala en su preámbulo «la dignidad intrínseca (...) de todos los miembros de la familia humana», para luego afirmar en su artículo $1^{\circ}$ que «todos los seres humanos nacen libres e iguales en dignidad y derechos» [7]. La Constitución Española de 1.978 señala en su artículo 10 que "la dignidad de la persona, los derechos inviolables que le son inherentes... son fundamento del orden político y de la paz social" [8].

En las últimas décadas, el concepto de dignidad humana ha adquirido especial relevancia en las cuestiones de bioética [9] destacando, sobre todo, la relación entre dignidad y final de la vida (muerte digna). En el contexto de los cuidados al final de la vida, considerando que la provisión de los mismos se da a personas próximas a morir, la salvaguarda de la dignidad de los pacientes cobra especial importancia, tanto para la sociedad en su conjunto como para los médicos en particular.

No existe una idea objetiva del término muerte digna. El carácter polisémico del mismo puede ser un problema en sí mismo cuando se habla de manejo médico del final de la vida y, en repetidas ocasiones, términos como el de cuidados paliativos, eutanasia o sedación se emplean como si fueran sinónimos del de muerte digna y, en consecuencia, se utilizan indistintamente, aunque sabemos que es un concepto mucho más amplio. El derecho a una muerte digna actualmente forma parte del 
lenguaje popular y es frecuente su inclusión en los medios de comunicación, pero no existe un derecho como tal a la muerte digna sino una serie de derechos relacionados: el derecho de los pacientes a declarar sus valores y preferencias, a recibir información, a participar en el proceso de toma de decisiones, a rechazar cualquier tratamiento aunque ello suponga un acortamiento de su vida, a recibir cuidados paliativos de calidad o a decidir acerca del cómo y el cuándo de su propia muerte. Desde hace décadas existen leyes y normas de carácter internacional, nacional y autonómico que enfatizan el carácter de derecho que tienen algunos de los términos anteriores. Estos derechos se consideran, en su mayoría, derechos condicionados en su titularidad [10], es decir se es titular de los mismos cuando una persona tiene una enfermedad avanzada o terminal o gravemente incapacitante y es dependiente del entramado médico. A su vez, estos derechos deben garantizar tres cuestiones: que un tratamiento médico no se prolongue contra la voluntad del paciente (encarnizamiento terapéutico), que en el proceso irremediable de su muerte, la persona no sufra innecesariamente, es decir, que pueda recibir unos buenos cuidados paliativos y su dolor sea tratado, incluyendo si es necesario una intervención para disminuir su nivel de conciencia (sedación terminal o paliativa) y ahora, con la regulación de la eutanasia poder decidir el cuándo y el cómo de su muerte cuando se den las condiciones precisas.

Tanto el concepto de muerte digna como el de dignidad cobran pleno sentido sobre la base de la autonomía de la persona. La dignidad y la libertad encuentran una base lógica en la autonomía propia del ser humano "...pues solo el que puede gobernarse a sí mismo es una persona libre o dicho de otra manera, una persona que obra de manera autónoma deja de estar al dictado de otras y, en consecuencia, actúa con libertad" [11].

Según normativa vigente [12] la autonomía personal se define como "la capacidad de controlar, afrontar y tomar, por propia iniciativa, decisiones personales acerca de cómo vivir de acuerdo con las normas y preferencias propias, así como de desarrollar las actividades básicas de la vida diaria".

El ejercicio de la autonomía plena precisa que la persona haya sido plenamente informada y sea capaz de expresar su consentimiento. En personas próximas a morir incapaces de expresar su voluntad, cuando es un tercero el que toma la decisión, cobra especial relevancia la declaración de voluntades anticipadas, el llamado testamento vital. Antes de la aprobación de la Ley de eutanasia, la legislación vigente en España ya incorporaba dos realidades: el derecho a declarar anticipadamente la voluntad y el derecho a recibir cuidados paliativos. Ambas quedan bien enmarcadas en la idea actual de muerte digna, porque el derecho a la autonomía de la voluntad remite a la teoría del consentimiento informado y el derecho a recibir cuidados paliativos abre la posibilidad de no alargar innecesariamente el sufrimiento, admitiendo el hecho de que pueden acortar la vida.

\section{Cuidados paliativos}

Los Cuidados Paliativos en España están regulados a nivel supranacional, nacional y autonómico. Las recomendaciones 1418 (1999) y 24 (2003) de la Asamblea Parlamentaria del Consejo de Europa de Protección de los Derechos Humanos y la Dignidad de los enfermos terminales y moribundos, plantean la necesidad de reconocer y proteger el derecho a los cuidados paliativos y señalan que es una responsabilidad de los gobiernos el garantizar que los cuidados paliativos sean accesibles a todos los que los necesiten [13].

La Organización Mundial de la Salud (OMS) ha venido reiterando la necesidad de desarrollar los cuidados paliativos como pilar fundamental para el alivio del sufrimiento y ha planteado la urgencia de que se facilite la disponibilidad de opioides y la mejora de la formación de los profesionales para el desarrollo de programas de cuidados paliativos [14]. La conferencia de expertos de la OMS reunida en Ginebra en 1990 definió los cuidados paliativos como "el cuidado total activo de los pacientes cuya enfermedad no responde a tratamiento curativo... el objetivo principal de los cuidados paliativos no es curar ni debe ser prolongar la vida, sino conseguir la mejor calidad de vida posible para el enfermo y su familia, donde el control del dolor y de otros síntomas y problemas psicológicos, sociales y espirituales es primordial". Esta definición incluía que los cuidados paliativos no debían limitarse a los últimos días de vida sino aplicarse progresivamente a medida que avanzaba la enfermedad y en función de las necesidades de pacientes y familias y señalaba que, aunque los 
cuidados paliativos se proporcionan fundamentalmente a pacientes oncológicos, era factible prestar este tipo de atención a pacientes con "enfermedades neurológicas, enfermedades pulmonares graves, insuficiencia cardiaca congestiva avanzada o SIDA" [15].

De acuerdo a esta definición y objetivos, lo que caracteriza a los cuidados paliativos es que:

1. Proporcionan alivio del dolor y otros síntomas

2. Afirman la vida, pero aceptan la muerte como algo inevitable

3. No intentan ni acelerar ni retrasar la muerte

4. Integran los aspectos espirituales y psicológicos en los cuidados del paciente

5. Ofrecen un sistema de soporte para ayudar a los pacientes a vivir tan activamente como sea posible hasta la muerte

6. Ofrecen un sistema de soporte para ayudar a las familias a adaptarse durante la enfermedad de los pacientes y en el duelo

Desde esta perspectiva, los principios básicos de los cuidados paliativos son la atención integral, individualizada y continuada, atendiendo a necesidades físicas, emocionales, sociales y espirituales de los enfermos; la consideración de la familia y el enfermo como unidad a tratar, intentando que la familia participe activamente en el cuidado del enfermo y la promoción de la autonomía y dignidad del enfermo definidas por él mismo.

Las recomendaciones de los expertos de la OMS han sido, y son, fundamentales porque han servido de inspiración y soporte de miles de programas de cuidados paliativos que se han desarrollado por todo el mundo. Desde un punto de vista organizativo y sea cual sea el modelo adoptado en un país, la OMS ha señalado de forma reiterada que los programas de cuidados paliativos totalmente desarrollados deberían incluir los siguientes componentes: 1) Atención domiciliaria, 2) Servicio de consulta con profesionales sanitarios expertos, 3) Centros de día y, 4) Cuidados en institución cerrada cuando sea necesario. Pero insisten en que ninguno de elementos anteriores es imprescindible para proporcionar buenos cuidados paliativos. Los tratamientos en estos programas deben ser rentables en términos de coste-efectividad, simples de administrar y fácilmente aplicables en el domicilio de los pacientes. En general, se recomienda adaptar la organización de los cuidados paliativos a la organización sanitaria de cada país, no creando estructuras alternativas.

En España, la Estrategia en Cuidados Paliativos del Sistema Nacional de Salud (SNS) [16], aprobada en marzo de 2007 identifica los pacientes a incluir en programas de cuidados paliativos y plantea un cambio de paradigma en los objetivos asistenciales, incluyendo un no rotundo al encarnizamiento terapéutico (considerado mala práctica clínica) y un objetivo claro centrado en el alivio del sufrimiento.

La Estrategia Nacional de Cuidados Paliativos asume como propios los principios de la OMS e incorpora lo que considera valores esenciales de los programas de cuidados paliativos [17]:

1. Derecho al alivio del sufrimiento

2. Valor intrínseco de cada persona como individuo autónomo y único

3. Calidad de vida definida por cada paciente

4. Expectativas del enfermo y familia sobre la respuesta del sistema sanitario a sus necesidades en el final de la vida

5. Solidaridad ante el sufrimiento

6. Acompañamiento a los pacientes y sus familias basado en sus necesidades

7. Atención integral, accesible y equitativa

8. Continuidad de la atención y coordinación de niveles asistenciales

9. Comunicación abierta y honesta

10. Reflexión ética y participación de los pacientes y sus familiares en la toma de decisiones

11. Competencia y desarrollo profesional continuados para responder adecuadamente a las necesidades de los pacientes y familiares 
12. Trabajo en equipo interdisciplinar

13. Actuaciones terapéuticas basadas en las mejores evidencias disponibles

14. No discriminación en función de la edad, sexo, ni de ninguna otra característica.

En España, hasta diez Comunidades Autonómicas tienen Leyes de muerte digna que recogen los Cuidados Paliativos como derecho. Por ejemplo, la Ley 4/2017 de Derechos y Garantías de la Dignidad de la Persona en el Proceso de Morir de la Comunidad Autónoma de Madrid [18] en su Artículo 11, referente al Derecho a los Cuidados Paliativos, al tratamiento del dolor y a la sedación paliativa señala:

“... todas las personas con enfermedad terminal tienen derecho a recibir, con

el máximo respeto a su dignidad personal y voluntad libremente expresada, cuidados paliativos integrales de calidad..."

Inicialmente los Cuidados Paliativos estuvieron dirigidos, sobre todo, a los pacientes oncológicos. Sin embargo, la tendencia de los últimos años está marcada por la inclusión en programas de cuidados paliativos de pacientes con enfermedades crónicas evolutivas de cualquier edad que se encuentren en situación avanzada y terminal, rompiendo lo que había sido uno de los componentes básico en la definición de pacientes a incluir en programas de cuidados paliativos como es la estimación de la supervivencia. Actualmente las intervenciones paliativas se basan fundamentalmente en las necesidades de los pacientes y familiares, más que en un plazo concreto de supervivencia esperada. La Estrategia Nacional de Cuidados Paliativos establece los siguientes criterios para la definición de los pacientes con enfermedad en fase avanzada y terminal:

15. Enfermedad incurable, avanzada y progresiva (pacientes con cáncer, pacientes con enfermedades crónicas evolutivas, pacientes infantiles).

Pronóstico de vida limitado

Escasa posibilidad de respuesta a tratamientos específicos

Evolución de carácter oscilante y frecuentes crisis de necesidades

Intenso impacto emocional y familiar

Repercusiones sobre la estructura cuidadora

Alta demanda y uso de recursos

\section{Sedación terminal}

En España todas las Leyes autonómicas de muerte digna comentadas en el epígrafe anterior recogen la sedación terminal o paliativa como un derecho:

“... todas las personas con enfermedad terminal tienen derecho a recibir, con el máximo respeto a su dignidad personal y voluntad libremente expresada, cuidados paliativos integrales de calidad, incluida la sedación paliativa si el dolor, o cualquier otro síntoma que produzca molestias severas, son refractarios al tratamiento específico, aunque ello implique un acortamiento de su vida. La administración de sedación paliativa deberá ajustarse a un procedimiento que contemple las circunstancias específicas de cada paciente y los métodos a utilizar según la situación clínica en cada caso" [18].

La sedación terminal o paliativa se define como “... la administración deliberada de fármacos en las dosis y combinaciones requeridas para reducir la conciencia de un paciente con enfermedad avanzada o terminal, tanto como sea preciso para aliviar adecuadamente uno o más síntomas refractarios, con su consentimiento explícito. Si este no es posible, se obtendrá de su representante. La sedación paliativa constituye la única estrategia eficaz para mitigar el sufrimiento ante la presencia de síntomas refractarios intolerables que no responden al esfuerzo terapéutico realizado en un periodo razonable de tiempo" [18]. 
La definición de sedación terminal incorpora términos como derecho, respeto, dignidad, voluntad libremente expresada, acortamiento de la vida, administración deliberada de fármacos, síntomas refractarios o estrategias eficaces para mitigar el sufrimiento, todos ellos términos comunes a los utilizados para hablar de la eutanasia y/o el suicidio medicamente asistido. Una de las principales diferencias estaría en la consideración de síntoma refractario, entendido como "... un síntoma que no puede ser adecuadamente controlado con los tratamientos disponibles, aplicados por médicos expertos, en un plazo de tiempo razonable. En estos casos el alivio del sufrimiento del enfermo requiere la sedación paliativa" [18]. A diferencia de lo que va a ocurrir cuando se plantee una solicitud de eutanasia, donde el propio paciente decide, en el caso de la sedación terminal es la situación clínica la que va a determinar su indicación.

No obstante, y dada la relevancia que tiene por su contribución al debate, transcribimos a continuación parte de la Declaración de Boston [1], elaborada tras el congreso de la Federación Mundial de Asociaciones pro-Derecho a Morir que se celebró en esa ciudad norteamericana en el año 2000:

“Apoyamos el derecho de las personas adultas capacitadas que sufren (...) a pedir ayuda médica para acelerar su muerte, si ése es su deseo racional, voluntario y reiterado, una vez que se les hayan ofrecido todas las opciones más adecuadas de medicina paliativa (...) queremos llamar la atención pública sobre la práctica de la «sedación terminal» o «eutanasia lenta», que se utiliza actualmente de manera muy extensa por todo el mundo en hospitales, residencias, unidades de cuidados paliativos y hogares particulares por la que un médico puede, legalmente, administrar dosis crecientes de analgésicos y sedantes que pueden acelerar la muerte de un paciente siempre que la intención declarada sea disminuir el dolor y el sufrimiento (...) creemos que la única diferencia real entre «sedación terminal» y una dosis letal de efecto rápido es sólo una cuestión de tiempos; una muerte lenta, que dura varios días, con medicación paliativa que acorta la vida, contra una muerte más digna y pacífica, porque no es prolongada y la decide el propio paciente. Instamos a otros profesionales médicos en todo el mundo, a ser más sinceros sobre esa forma de muerte médicamente asistida".

Señalábamos en la introducción de este trabajo que hay un aspecto controvertido en la relación entre los cuidados paliativos y la eutanasia relativo a si son conceptos complementarios o antagónicos. Señalar, antes de entrar en la conceptualización y definiciones de eutanasia y SMA que en los países donde se han legalizado estas prácticas, los cuidados paliativos han experimentado un importante impulso, no como estrategia contrapuesta sino complementaria. Países como Bélgica, que tiene legalizada la eutanasia, o EEUU que tiene legalizado el SMA exigen la inclusión, o la posibilidad de inclusión, en programas de cuidados paliativos para acceder a las leyes de eutanasia.

\section{Eutanasia y suicidio medicamente asistido}

La eutanasia viene de los vocablos griegos thánatos que significa muerte y del prefijo eu que significa bueno o fácil. Así entendido, eutanasia simplemente se refiere a una buena muerte, a una muerte fácil. Expresado de esta manera, el termino eutanasia es completamente inocuo y, a priori, cualquiera pediría una muerte todo lo buena y fácil que pueda ser. A mediados del siglo XX, el concepto de eutanasia cambia y es cada vez más utilizado en su acepción de alternativa para proporcionar una muerte intencionada.

Tradicionalmente, la eutanasia se ha dividido en pasiva (o negativa) y activa (o positiva). La activa, a su vez, se divide en directa e indirecta. Aunque también puede clasificarse como voluntaria, no voluntaria e involuntaria, existe acuerdo actualmente en limitar el empleo del término eutanasia a aquella intervención que se produce de manera activa y directa. En consecuencia, cuando se utilice en este trabajo el término eutanasia, nos estaremos ajustando estrictamente a esa definición.

La eutanasia presupone la intervención de un agente externo, específicamente un médico, que administra a un paciente una sustancia en la dosis y por la vía necesaria con la intención de provocar una muerte rápida e indolora [2] y, cuestión clave, la petición de eutanasia la hace el propio paciente 
(eutanasia voluntaria) o su representante legal si el paciente no puede decidir (eutanasia novoluntaria).

Si el médico únicamente prescribe una dosis letal de un medicamento para que se lo autoadministre el paciente, ya no hablamos de eutanasia e introducimos el concepto de Suicidio Medicamente Asistido (SMA) [2].

Hay que destacar la diferencia entre administrar y prescribir, que es clave para diferenciar eutanasia y SMA. Aunque es frecuente que en la literatura aparezcan como términos intercambiables, enmarcados en el llamado contexto eutanásico, tienen diferencias éticas y jurídicas significativas, al menos desde la perspectiva internacional. En EEUU las leyes estatales que han regulado el SMA lo hacen con el propósito ético de que ni los médicos ni cualquier otra persona que participe en el proceso se consideren responsables de la muerte de los pacientes [19]. Según las leyes de EEUU si el paciente no puede autoadministrarse la medicación que provocará su muerte, nadie más puede hacerlo.

Aunque sea reiterativo, el Tribunal Europeo de Derechos Humanos, en su sentencia de 14 de mayo de 2013 (caso Gross vs. Suiza) define muy bien la eutanasia y el SMA [20]. En dicho fallo, el Tribunal con sede en Estrasburgo, resolvió la petición de suicidio asistido de Alda Gross, una anciana suiza que no padeciendo ninguna enfermedad terminal pero que no soportaba el deterioro físico de su cuerpo, pidió una dosis letal de fenobarbital. La sentencia, que salió adelante con tres votos a favor y dos en contra, consideró la falta de directrices europeas para que ciudadanos sanos pudieran ser asistidos en su deseo de morir y distingue entre dos conductas eutanásicas diferentes:

“...la eutanasia activa y aquella en la que es el propio o la propia paciente la persona que termina con su vida, para lo que precisa de la colaboración de un profesional sanitario que, de forma intencionada y con conocimiento, facilite los medios necesarios, incluido el asesoramiento sobre la sustancia y dosis necesarias de medicamentos, su prescripción, o, incluso, su suministro con el fin de que él o la paciente se lo administre. Por su parte, eutanasia activa es la acción por la que un profesional sanitario pone fin a la vida de un o una paciente de manera deliberada y a petición de éste o ésta, cuando se produce dentro de un contexto eutanásico por causa de enfermedad grave e incurable o discapacidad grave crónica causantes de un sufrimiento intolerable".

Los términos eutanasia y SMA a menudo son sustituidos por sinónimos y metáforas, contribuyendo a una cierta confusión terminológica. En la Ley española, el término eutanasia aparece solo en el título y en el preámbulo, pero la palaba suicidio no aparece en parte alguna del documento.

A diferencia de lo que ocurre con los cuidados paliativos, la experiencia internacional en relación a la eutanasia es muy escasa. Apenas una decena de países en todo el mundo han avanzado en la regulación de esta cuestión y existen profundas diferencias tanto en los antecedentes como en la regulación misma de aquellos que si la tienen. Hasta la aprobación de la Ley española, en la Unión Europea de los 27, con una población de 447 millones de personas [21] tres de sus Estados miembros (Bélgica, Holanda y Luxemburgo) tenían legalizada la eutanasia y/o el SMA. En números, 29 millones de ciudadanos que representan el 6,4 \%. Ahora, con España, este porcentaje es del 17\%. Portugal ha aprobado en 2021 una Ley de eutanasia, actualmente paralizada por su Tribunal Constitucional.

La regulación de la eutanasia es un fenómeno nuevo y con poca implantación, destacando la heterogeneidad en los países en los que se ha legalizado. Sirva como ejemplo que las leyes de Oregón [19], de Holanda [22] y de Bélgica [23], difieren en aspectos esenciales: la de Oregón permite el SMA pero prohíbe explícitamente la eutanasia; la Ley holandesa permite ambas alternativas y la Ley belga solo regula la eutanasia. En ninguna de estas dos leyes se reconoce la eutanasia/SMA como derecho.

La Ley española que la regula la eutanasia y el SMA es una recién nacida pendiente de desarrollo en la que hay dos aspectos muy relevantes: se reconoce el derecho de toda persona que cumpla los requisitos previstos a solicitar y recibir la prestación de ayuda para morir (Cap. II, Articulo 4) y no dice nada sobre la expectativa de vida del paciente. No es necesario que el paciente sufra una enfermedad que amenace su vida porque la Ley no excluye que se atienda una petición de eutanasia de un paciente que todavía pueda vivir muchos años. Y estas son dos grandes diferencias con otros tratamientos al final de la vida, como los cuidados paliativos y la sedación terminal. Lo que no es fácil 
de determinar es el desarrollo que va a tener una Ley tan nueva y controvertida. De cara al papel que los médicos van a tener en la implementación de la Ley, se puede aprovechar la similitud entre las líneas principales de las Leyes de los Países Bajos [24] y de España [25] y revisar los requisitos de diligencia y cuidados que implican a un médico holandés cuando recibe una solicitud de eutanasia:

1. Tiene que estar convencido de que la petición de eutanasia de sus pacientes es voluntaria y reiterada

2. Tiene que estar convencido de que existe un sufrimiento insoportable y sin perspectivas de mejora

3. Tiene que haber informado al paciente sobre la situación en que se encuentra y sus perspectivas de futuro

4. Tiene que haber llegado, junto con el paciente, al convencimiento de que no existe otra solución razonable en la que se encuentra el paciente

5. Tiene que haber consultado con otro médico independiente

6. Tiene que haber llevado a cabo la terminación de la vida con la mayor diligencia y cuidado

En cuanto a la petición voluntaria tiene que diferenciar entre: a) Voluntariedad externa, que implica que no haya habido influencia perceptible de otros $y, b$ ) voluntariedad interna, según la cual el paciente debe tener capacidad para expresar su voluntad y debe comprender la información (médica) relevante. El paciente debe estar en condiciones de explicar claramente por qué desea la eutanasia. Una situación especial que puede generar conflicto es la solicitud de pacientes con enfermedad mental. En Holanda, de hecho, es necesario que expertos valoren cuidadosamente la voluntariedad, descartando que haya podido estar afectada por la enfermedad mental.

En cuanto a la petición reiterada no puede tratarse de una petición hecha en el marco de un impulso repentino. La repetición (constante) de la petición es un factor a tener muy en cuenta. En Holanda se considera que se dan circunstancias especiales en los siguientes casos: pacientes con enfermedad mental, con demencia, con discapacidad intelectual, pacientes en coma o con disminución del nivel de conciencia y menores de edad.

La consideración del sufrimiento insoportable merece siempre especial atención. Ya hemos señalado anteriormente que el sufrimiento puede ser consecuencia del dolor y la ansiedad, del agotamiento y el cansancio, del deterioro físico, de la ausencia de perspectivas de mejora, pero también de una dependencia creciente, de un deterioro experimentado por el paciente, o de la perdida de dignidad. El médico debe sentir y comprender el sentimiento concreto de un paciente concreto ("Ponerse en el lugar del otro"). Una de las sentencias holandesas (Brongersma, 2002) señala que "...el sufrimiento del paciente debe provenir fundamentalmente de una o más enfermedades o afecciones clasificadas medicamente". Es decir, el sufrimiento debe tener un FUNDAMENTO MÉDICO, entrar en el dominio del médico. En consecuencia, el concepto de "vida agotada" (o "cansancio vital") no entra en el campo de acción de la ley holandesa.

Cuando una persona le comunica que su vida ya no tiene sentido, que su vida está agotada y que su sufrimiento no tiene consuelo (bien por sentimientos de inutilidad o de dependencia o porque la pérdida de control es vivida por ella como intolerable para su idea de dignidad) el papel del médico cambia porque es cuestionable que esos problemas puedan ser solucionados adecuadamente por la medicina.

A efectos prácticos, para pedir eutanasia en Holanda es necesario que el paciente experimente el sufrimiento. Pacientes con disminución del nivel de conciencia o en coma, no pueden acceder a una petición de eutanasia. El hecho de que los allegados del paciente consideren la situación como denigrante, no tiene influencia alguna.

El paciente que solicita una eutanasia recibe información completa sobre su situación y sobre sus perspectivas y el médico tiene que constatar que el paciente ha comprendido toda esa información.

Finalmente, un médico en Holanda que recibe una solicitud de eutanasia tiene que haber llegado, junto con el paciente, al convencimiento de que no existe otra solución razonable en la que 
se encuentra el paciente. Se considera que existe una alternativa razonable para la eutanasia si hay algún camino real para aliviar o eliminar el sufrimiento del paciente, considerando que el paciente está en su derecho de rechazar un tratamiento. Por ejemplo, si el sufrimiento del paciente va acompañado de dolor, el tratamiento analgésico podría ser una alternativa para la eutanasia.

\section{Algunas reflexiones}

En el conjunto de los tratamientos al final de la vida de los pacientes, los cuidados paliativos y la sedación ya forman parte de la práctica habitual y están incluidos en la Cartera de Servicios del SNS. Los planes y las estrategias de cuidados paliativos siguen planteando mejoras en la definición de enfermos a incluir (oncológicos y no oncológicos) en los diferentes programas, en cómo encajar de manera eficiente esos programas en el propio sistema de salud o estableciendo el papel de las unidades de cuidados paliativos en un sistema nacional de salud organizado en dos niveles asistenciales.

En cuanto a la eutanasia y el SMA, los médicos, en cuantos ejecutores de esta práctica, serán actores clave en esta cuestión. Las instituciones que representan formalmente a los profesionales (Colegios Profesionales de medicina y enfermería, Organización Médica Colegial, Asociación Médica Mundial, etc.) [26] han lanzado un mensaje de rechazo de la Ley por considerar que la eutanasia no es un acto médico. La influencia que estos mensajes puedan tener sobre los médicos individualmente es desconocida. La objeción de conciencia está contemplada en la Ley ya que si bien el Estado debe garantizar el derecho a que una persona pueda morir mediante la aplicación de la eutanasia o el suicidio asistido, también debe garantizar el derecho de los médicos a objetar de acuerdo a su conciencia.

Como ya se ha comentado en otra parte del trabajo existe un aspecto que levanta enorme controversia y es la relación entre los cuidados paliativos y la eutanasia. Para los que están en contra de la Ley, los cuidados paliativos hacen innecesaria la regulación de la eutanasia porque consideran que los mismos satisfacen todas las necesidades que presentan los pacientes próximos a morir. Llegan a señalar que los cuidados paliativos vendrían a ser una especie de "antídoto" de la eutanasia que eliminaría el conflicto moral que subyace en la misma. Para los que están a favor de la Ley, los cuidados paliativos serían un derecho "complementario" al de eutanasia. No existen datos que permitan adelantar cuantas personas van a solicitar ayuda para morir. Nuestra impresión después de años de actividad asistencial con pacientes en fase terminal próximos a morir es que las solicitudes de ayuda a morir que se han recibido, y que por razones evidentes no se pudieron atender, no han sido de pacientes con dolor incontrolado, ansiedad extrema o ahogo intenso. Más bien se producían en pacientes bien controlados desde el punto de vista sintomático, conocedores de su situación y de su mal pronóstico a corto plazo, que consideraban que su vida no merecía la pena ser vivida y, en consecuencia, pedían ayuda para morir. Es decir, primaba el componente de complementariedad frente al de antagonismo.

Finalmente, señalar que en España no ha habido una reflexión colectiva y un debate real sobre la eutanasia. Tampoco lo ha habido en relación a la muerte en sí misma. Llevamos décadas repitiendo una idea acuñada hace tiempo por pensadores de diferentes sitios y culturas y un millón de veces repetida: la sociedad vive de espaldas a la muerte. Ahora podríamos decir que la sociedad, y los médicos, viven de espaldas a la muerte porque, como sugieren esos autores, para la sociedad, y los propios médicos, la muerte es un tabú. El pensador y filósofo español J.L. Aranguren, en un artículo publicado en 1990 [27], antes del boom tecnomédico, decía que “...hoy solo sentimos la senilidad cuando se hace valetudinaria, requiere asistencia, padece enfermedad crónica y, por tanto, incurable. Esta enfermedad llega a aparecer como terminal y entonces la muerte que, hasta ese momento, gracias al tabú de la muerte, no se nos había aparecido sino como muerte «de los otros» y, a lo sumo, como pasajera «angustia ante la muerte», ahora está ahí, al acecho, esperando caer sobre mí".

Es muy probable que la mayoría de las personas que mueren en el mundo occidental van a estar en esa situación y pueden adoptar distintas posiciones. Algunas, desde posiciones fuertemente religiosas, pueden vivir el final de su vida sin ansiedad alguna, con la tranquilidad de un tránsito a una vida mejor. No se puede saber cuántas personas están en esta situación, pero lo que casi se puede 
afirmar es que no van a solicitar ayuda para morir. Un segundo grupo de personas estaría integrado por aquellos que luchan hasta el final, instaladas en una negación que les va a llevar a esa lucha constante para no morir. Probablemente va a ser el grupo más numeroso porque, entre otras cosas, el paradigma médico actual anima a esa lucha desigual. Finalmente habría un grupo de personas que, suficientemente informadas y conocedoras de su situación, van a aceptar que están al final de su vida. $\mathrm{Y}$ en este último grupo, sabemos que una parte esperará el final con buenos cuidados paliativos y control del dolor. Pero otra parte, demandará ayuda para morir, en la convicción de que esa etapa final de sus vidas no merece la pena ser vivida. Para estas personas, muchas o pocas, la Ley actual que regula la eutanasia es necesaria.

\section{Referencias Bibliográficas}

1. The Boston Declaration on Assisted Dying. 3th International Conference of the World Federation of Right to Die Societies held in Boston, Massachusetts from September 1 to 3, 2000

2. Ley Orgánica 3/2021, de 24 de marzo sobre regulación de la eutanasia. Ministerio de Sanidad. BOE $n^{\circ} 72$ de 25 de marzo de 2021

3. Instituto Nacional de estadística (INE). Datos demográficos básicos. Madrid: INE, 2021. Disponible en https://www.ine.es/dyngs/INEbase/es/operacion.htm

4. Callahan D. The troubled dream of life. In search of a peaceful death. Simon and Schuster. New York: 1993

5. Instituto Nacional de estadística. Tablas de mortalidad por causa (Internet). INE: Madrid, 2020

6. Ley 41/2002 de 14 de noviembre, Básica Reguladora de la Autonomía del Paciente. BOE núm. 274 de 15 de noviembre de 2002

7. Asamblea General de la Organización de Naciones Unidas (1948). Declaración Universal de Derechos $\begin{array}{lllll}\text { Humano } & \text { (217 } & \text { [III] } & \text { A) } & \text { Paris. }\end{array}$ https://www.ohchr.org/en/udhr/documents/udhr_translations/spn.pdf

8. Constitución Española. Boletín Oficial del Estado, 29 de diciembre de 1978, núm. 311, p. 29313 a 29424

9. Adorno R. Human Dignity and human rights as a common ground for a global bioethics. Journal of Medicine and Philosophy 2009, vol. 34, nª 3, p. 223-240)

10. Pérez Miras A. Muerte digna y estatutos de autonomía. Derecho y Salud vol. 25, no extra 1, 2015, p. 96-104

11. KANT, I. Fundamentación de la metafísica de las costumbres, trad. M. García Morente, Espasa-Calpe, Madrid, 1980

12. Ley 39/2006 de 14 de diciembre de Promoción de la Autonomía Personal y Atención a las Personas en Situación de Dependencia. BOE no 299 de 15 de diciembre de 2006

13. Asamblea Parlamentaria del Consejo de Europa. Recomendación 1418 (1999). Protección de los derechos humanos y la dignidad de los enfermos terminales y moribundos. Adoptada el 25 de junio de 1999. Disponible en http://www.unav.es/cdb/acoerec99-1418.html

14. Organización Mundial de la Salud. Cancer pain relief and palliative care. Report of a WHO Expert Committee (WHO Technical Report Series, No. 804). Ginebra: OMS, 1990

15. Organización Mundial de la Salud. Cancer pain relief and palliative care. Ginebra: WHO, 1994. Technical Report Series: 804

16. Estrategia en Cuidados Paliativos del Sistema Nacional de Salud. Buenas prácticas 2007-2009. Madrid: Ministerio de Sanidad, Política Social e Igualdad, 2010

17. http://www.msc.es/organizacion/sns/planCalidadSNS/pdf/excelencia/ diabetes/CUIDADOS_PALIATIVOS/estrategiaCuidadosPaliativos.pdf

18. Ley 4/2017, de 9 de marzo, de Derechos y Garantías de las Personas en el Proceso de Morir. B.O.C.M nº 69 de 22 de marzo de 2017

19. Oregon Death with Dignity Act, $1998 . \quad$ Disponible en: https://www.oregonlegislature.gov/bills_laws/ors/ors127.html

20. Caso de Gross vs. Suiza. Tribunal Europeo de Derechos Humanos, caso no ${ }^{\circ}$ 67810/10, 2013

21. Eustat. Población en la UE 27 en 2020 (no de habitantes)

22. Holanda. Ley 26691/2001 sobre La Terminación de la Vida a Petición Propia y del Auxilio al suicidio. Ministerio de Justicia. Ministerio de Sanidad, Bienestar y Deporte

23. Bélgica. Belgian Official Collection of the Laws. The Belgium Act on Euthanasia of May, 28th 2002

24. KNMG/KNMP. Guidelines for the Practice of Eutanasia and Pysician-Assisted Suicide. August 2012 
25. Ministerio de Sanidad. Manual de Buenas Prácticas en Eutanasia. Ley Orgánica 3/2021, de 24 de marzo, de regulación de la eutanasia. Madrid: 2021

26. El CGCOM ante la aprobación en el Congreso de los Diputados del dictamen de la Comisión de Justicia obre la Proposición de Ley Orgánica para la regulación de la eutanasia en España. Disponible en: https://www.cgcom.es/sites/default/files/u183/np_eutanasia_21_05_18.pdf

27. Aranguren, Calidad de vida, Calidad de muerte. Quadern CAPS, nov 1990, no 14

(C) 2021 por los autores; Esta obra está sujeta a la licencia de Reconocimiento 4.0 Internacional de Creative Commons. Para ver una copia de esta licencia, visite http://creativecommons.org/licenses/by-nc-nd/4.0/. 